\title{
Development and external validation of nomograms to predict the risk of skeletal metastasis at the time of diagnosis and skeletal metastasis-free survival in nasopharyngeal carcinoma
}

Lin Yang ${ }^{1,2,3+}$ (D), Liangping Xia ${ }^{1,2,3+}$, Yan Wang ${ }^{1,2,3 \dagger}$, Shasha He ${ }^{1,2,3}$, Haiyang Chen ${ }^{4}$, Shaobo Liang ${ }^{5}$, Peijian Peng ${ }^{6}$, Shaodong Hong ${ }^{1,2,3^{*}}$ and Yong Chen ${ }^{1,2,3^{*}}$

\begin{abstract}
Background: The skeletal system is the most common site of distant metastasis in nasopharyngeal carcinoma (NPC); various prognostic factors have been reported for skeletal metastasis, though most studies have focused on a single factor. We aimed to establish nomograms to effectively predict skeletal metastasis at initial diagnosis (SMAD) and skeletal metastasis-free survival (SMFS) in NPC.

Methods: A total of 2685 patients with NPC who received bone scintigraphy (BS) and/or 18F-deoxyglucose positron emission tomography/computed tomography (18F-FDG PET/CT) and 2496 patients without skeletal metastasis were retrospectively assessed to develop individual nomograms for SMAD and SMFS. The models were validated externally using separate cohorts of 1329 and 1231 patients treated at two other institutions.

Results: Five independent prognostic factors were included in each nomogram. The SMAD nomogram had a significantly higher $\mathrm{c}$-index than the TNM staging system (training cohort, $P=0.005$; validation cohort, $P<0.001$ ). The SMFS nomogram had significantly higher $c$-index values in the training and validation sets than the TNM staging system $(P<0.001$ and $P=0.005$, respectively). Three proposed risk stratification groups were created using the nomograms, and enabled significant discrimination of SMFS for each risk group.
\end{abstract}

Conclusion: The prognostic nomograms established in this study enable accurate stratification of distinct risk groups for skeletal metastasis, which may improve counseling and facilitate individualized management of patients with NPC.

Keywords: Nasopharyngeal carcinoma, Skeletal metastasis at the time of diagnosis (SMAD), Skeletal metastasis free survival (SMFS), Prognosis, Nomograms

\footnotetext{
* Correspondence: hongshd@sysucc.org.cn; chenyong@sysucc.org.cn

${ }^{\dagger}$ Equal contributors

'Sun Yat-sen University Cancer Center, 651 East Dong Feng Road,

Guangzhou 510060, China

Full list of author information is available at the end of the article
} 


\section{Background}

Nasopharyngeal carcinoma (NPC) is a malignant head and neck cancer with a distinct ethnic and geographic pattern of distribution; the highest incidences of NPC (30-80 cases per 10,000/year) are observed in southern China and South East Asia [1]. Developments in advanced imaging modalities and instrumentation have enabled more precise tumor staging. Currently, approximately $5-8 \%$ of cases of NPC have distant metastasis (M1) at first diagnosis; the skeleton is the most common distant metastasis site, representing $70 \%$ to $80 \%$ cases of M1 disease [2-4]. Distant metastasis at diagnosis is associated with poorer survival outcomes and reduced quality of life. Moreover, research on $\mathrm{M} 1$ disease is sparse due to the poor survival outcomes of patients with skeletal metastases. However, increasing evidence indicates long-term survival and even a complete response can be achieved among a small proportion of patients with skeletal metastases, especially those who receive aggressive treatment [5]. This indicates different treatment methods could significantly improve the prognosis of selected high-risk M1 cases. However, solely relying on the TNM classification to predict the outcomes of patients with skeletal metastasis may result in inaccurate assessment, leading to unnecessary treatment and financial burdens or - even worse - the patient receiving a suboptimal treatment strategy. Moreover, individualized follow-up and treatment strategies may be required for specific subgroups of patients with different risks of skeletal metastasis.

Bone scintigraphy (BS) remains is the leading diagnostic method for bone metastasis during initial work-up as it is widely available and low cost. However, BS is not routinely conducted during follow-up as it has a low diagnostic sensitivity, especially for early bone metastatic lesions; metastases mainly located in the bone marrow are frequently not detected by BS [6]. Although 18F-FDG PET/ CT has a higher sensitivity than BS for detecting bone metastases in primary NPC, 18F-FDG PET/CT technique is expensive [7]. However, differentiation of malignant and benign lesions on BS and 18F-FDG PET remains problematic, even for experienced nuclear physicians.

As far as we are aware, research on the frequency of bone metastases at initial diagnosis (SMAD) and skeletal metastasis-free survival (SMFS) in NPC is rare and narrowly-focused [8-11]. The lack of such data hampers accurate patient staging and risk stratification and delays the design of more reliable treatment protocols, as the M1 category is a "catch-all" classification that includes patients whose treatment response could be potentially curable or incurable. Identifying subgroups of patients with different risks of bone metastasis could help determine the appropriate imaging techniques and follow-up timing in a more personalized manner. Furthermore, more accurate prediction of the risk of skeletal metastasis could provide valuable decision-making information for clinicians and patients.
Nomograms incorporate a variety of important factors and have been demonstrated to be reliable prediction tools for quantifying individual risk in cancer. Nomograms can provide more precise prognoses than the traditional TNM staging system in several tumor types. To date, there has been no attempt to establish nomograms to predict SMAD and SMFS in NPC. We hypothesized nomograms combining $\mathrm{T}$ category, $\mathrm{N}$ category and other objective laboratory indexes could generate more accurate predictive models for SMAD and SMFS. Therefore, we assessed the prognostic risk factors for SMAD and SMFS in a large cohort of patients with NPC and validated the resulting nomograms using an external cohort treated at two other institutions.

\section{Methods}

\section{Training cohort}

The training cohort was derived from patients treated at Sun Yat-sen University Cancer Center between and December, 2012. The inclusion criteria were: (i) pathologically confirmed NPC; (ii) complete pretreatment clinical information and laboratory data; (iii) BS and/or 18F-FDG PET/CT at diagnosis of NPC; and (iv) complete follow-up data. Exclusion criteria were incomplete followup data, death due to non-NPC-associated accident, or previous/synchronous malignant tumors. Ethical approval was obtained from the institutional review boards. The requirement for informed consent was waived as this was a retrospective study. The study protocol complied with the Declaration of Helsinki and was approved by the Ethics Committee of Sun Yat-sen University Cancer Center.

A standardized form was designed to retrieve all relevant data, including sociodemographic data (age, gender, smoking history, alcohol exposure, family history of malignant tumors, family history of NPC); baseline laboratory data including plasma Epstein-Barr virus (EBV) DNA copy number, serum calcium, serum magnesium, serum phosphorus, serum albumin(ALB), serum globulin (GLB), serum aspartate transaminase (AST), serum alanine transaminase (ALT), serum alkaline phosphatase (ALP), serum lactate dehydrogenase (LDH), serum C-reactive protein (CRP); T category [primary tumor location, size, extension], $\mathrm{N}$ category [number/location of lymph node metastases); and treatment data (radiotherapy technique, fractions, dosage; chemotherapy). Clinical stage was assessed using the seventh edition of the AJCC/ UICC TNM staging system.

\section{Treatment}

All patients were treated using definitive radiotherapy (RT). The dose ranges for the nasopharynx, node-positive region and node-negative regions were 60-80, 60-70, and 50-60 Gy, respectively. Patients with stage I or II NPC did not receive chemotherapy; patients with stage III or IV 
NPC received induction, concurrent or adjuvant chemotherapy (or a combination of these strategies) as recommended by the institutional guidelines. Induction or adjuvant chemotherapy were cisplatin with 5-fluorouracil; cisplatin with taxoids; or cisplatin, 5-fluorouracil and taxoids (every 3 weeks; two to three cycles). Concurrent chemotherapy was cisplatin in weeks 1,4 and 7 of radiotherapy or cisplatin weekly.

\section{Validation cohort}

To examine the general applicability of the model, an independent external validation cohort of 1329 consecutive patients with NPC who received definitive radiotherapy at the Fifth affiliated hospital of Sun-Yat Sen University and the First hospital of the Foshan between January, 2006 and December, 2012 were included. Inclusion and exclusion were the same as the training cohort. Sufficient data was available for all patients to score all variables in the nomograms established in this study.

\section{Statistical analysis}

SMAD was defined as the presence of skeletal metastasis on BS or 18F-FDG PET/CT at initial diagnosis (before receiving any treatment). SMFS was measured as time from diagnosis to detection of skeletal metastasis or censorship at last follow-up. In the training set, continuous variables were expressed as mean ( \pm standard deviation), medians and ranges were transformed into dichotomous variables using the median value. Categorical variables were compared using the chi-square test or Fisher's exact test; categorical/continuous variables, univariate logistic regression. Variables achieving significance at the level of $P<0.05$ were entered into multivariate logistic regression analyses via stepwise procedures. In the training set, survival curves for different variables were plotted using the Kaplan-Meier method and compared using the logrank test. Significant variables $(P<0.05)$ were entered into the Cox proportional hazards multivariate analyses to identify independent prognostic factors via forward stepwise procedures $(P<0.05)$. Statistical data analyses were performed using SPSS 22.0 (SPSS, Chicago, IL, USA).

Based on multivariate analyses, nomograms were generated to provide visualized risk prediction using the survival and rms packages of R 2.14.1 (http://www.r-project.org). Nomograms were subjected to bootstrap resampling $(n=1000)$ for interval and external validation to correct the concordance index (c-index) and explain variance with respect to over-optimism. The ability of the nomograms and TNM staging system to predict survival were compared using the c-index, a variable equivalent to the area under curve (AUC) of receiver operating characteristic curves for censored data. The maximum c-index value is 1.0 , which indicates perfect prediction, while 0.5 indicates the probability of correctly predicting the outcomes by random chance. The nomogram and TNM staging system were compared using rcorrp.cens in the Hmisc module of R. The nomogram for 1-, 3-, and 5-year SMFS was calibrated by comparing predicted and actual observed survival rates. During external validation, the nomogram point scores were calculated for individual patients, then Cox regression analysis was performed using total point scores as a predictor in the validation cohort.

In addition to numerically comparing discriminative ability by c-index, we also attempted to confirm the superior independent discriminative ability of the nomograms over the standard TNM staging system. The training cohort were evenly grouped into three risk groups by nomogram score, then we investigated the predictive ability of the risk stratification cut-off points and different subgroups (TNM stage) using Kaplan-Meier survival curve analysis. A two-sided $P$ value $<0.05$ was deemed significant. Details of the $\mathrm{R}$ code used to generate the nomograms can be assessed in the additional information online (Additional file 1). This trial was registered with Clinical Trials.Gov (NCT00705627); all data has been deposited at Sun Yat-sen University Cancer Center for future reference (number RDD RDDA2017000293).

\section{Results}

\section{Patient characteristics and survival}

A total of 2685 and 1329 patients in the training and external validation cohorts were eligible for the SMAD analyses (Additional file 2: Figure S1). Median age was 45-years-old (range, 23 to 78-years-old) for the training cohort and 45-years-old (range, 19 to 70-years-old) for the validation cohort. After excluding patients with distant metastasis at diagnosis, 2469 and 1231 patients were included in the analyses for SMFS. Median followup for SMFS in the training cohort was 65.0 months and 61.8 months in the validation cohort. Five-year SMFS was $86 \%$ in the training cohort and $85.4 .0 \%$ in the validation cohort. In both cohorts, a total of 391 patients (9.7\%) developed skeletal metastases after initial diagnosis, and 287 patients (7.7\%) were confirmed to have skeletal metastases at initial diagnosis. The characteristics of the cohorts are summarized in Table 1 and Additional file 3: Table S1.

\section{Univariate and multivariate analyses}

The factors associated with significantly poorer SMAD included in the univariate logistic regression model were sex (male); elevated LDH, CRP, ALP, platelets, monocytes, neutrophils and plasma EBV DNA; decreased hemoglobin (HGB) and ALB; and advanced clinical N category. All significant variables were entered into multivariate logistic regression; ALP, LDH, HGB, plasma EBV DNA and N category retained independent prognostic significance for SMAD. 
Table 1 Associations between the clinical and laboratory characteristics of the patients and SMAD as indicated by the chi-square test or Fisher's exact test

\begin{tabular}{|c|c|c|c|c|c|}
\hline \multirow[t]{3}{*}{ Characteristic } & \multirow[t]{3}{*}{ Number (\%) } & \multirow{2}{*}{\multicolumn{2}{|c|}{ Training cohort }} & \multirow[t]{3}{*}{$P$-value } & \multirow{3}{*}{$\begin{array}{l}\text { Validation cohort } \\
\text { Number (\%) }\end{array}$} \\
\hline & & & & & \\
\hline & & Absent & Present & & \\
\hline \multicolumn{4}{|l|}{ Age, years } & 0.379 & \\
\hline$<45$ & $1404(52.3 \%)$ & 1311 (93.4\%) & $93(6.6 \%)$ & & 679 (51.1\%) \\
\hline$\geq 45$ & $1281(47.7 \%)$ & 1185 (92.5\%) & $96(7.5 \%)$ & & $650(48.9 \%)$ \\
\hline \multicolumn{4}{|l|}{ Sex } & 0.025 & \\
\hline Male & $2131(79.4 \%)$ & 1969 (92.4\%) & $162(7.6 \%)$ & & 986 (74.2\%) \\
\hline Female & $554(20.6 \%)$ & 527 (95.1\%) & $27(4.9 \%)$ & & $343(25.8 \%)$ \\
\hline \multicolumn{4}{|l|}{ Smoking Status } & 0.055 & \\
\hline Absent & 1708 (63.3\%) & 1600 (93.7\%) & $108(6.3 \%)$ & & 795 (59.8\%) \\
\hline Present & 977 (36.4\%) & 896 (91.7\%) & $81(8.3 \%)$ & & 534 (40.2\%) \\
\hline \multicolumn{4}{|l|}{ Drinking Status } & 0.873 & \\
\hline Absent & $2382(88.7 \%)$ & 2215 (93.0\%) & $167(7.0 \%)$ & & $1117(84.0 \%)$ \\
\hline Present & $303(11.3 \%)$ & $281(92.7 \%)$ & $22(7.3 \%)$ & & $212(16.0 \%)$ \\
\hline \multicolumn{4}{|l|}{ Family history } & 0.566 & \\
\hline Absent & 1926 (71.7\%) & 1787 (92.8\%) & $139(7.2 \%)$ & & 967 (72.8\%) \\
\hline Present & 759 (28.3\%) & 709 (93.4\%) & $50(6.6 \%)$ & & $362(27.2 \%)$ \\
\hline \multicolumn{4}{|l|}{ Calcium, mmol/L } & 0.932 & \\
\hline$<2.4$ & 1370 (51.0\%) & 1273 (92.9\%) & 97 (7.1\%) & & $501(37.7 \%)$ \\
\hline$\geq 2.4$ & 1315 (49.0\%) & $1223(93.0 \%)$ & $92(7.0 \%)$ & & $828(62.3 \%)$ \\
\hline \multicolumn{4}{|l|}{ Phosphorus, mmol/L } & 0.587 & \\
\hline$<1.15$ & 1398 (52.1\%) & $1296(92.7 \%)$ & $102(7.3 \%)$ & & 676 (50.9\%) \\
\hline$\geq 1.15$ & 1287 (47.9\%) & 1200 (93.2\%) & 87 (6.8\%) & & 653 (49.1\%) \\
\hline \multicolumn{4}{|l|}{ Magnesium, mmol/L } & 0.308 & \\
\hline$<0.93$ & 1410 (52.2\%) & 1304 (92.5\%) & $106(7.5 \%)$ & & 919 (69.1\%) \\
\hline$\geq 0.93$ & 1275 (47.5\%) & 1192 (93.5\%) & $83(6.5 \%)$ & & 410 (30.9\%) \\
\hline \multicolumn{4}{|l|}{$C R P, m g / L$} & $<0.001$ & \\
\hline$<1.91$ & 1345 (50.1\%) & 1283 (95.4\%) & $62(4.6 \%)$ & & 722 (54.3\%) \\
\hline$\geq 1.91$ & 1340 (49.9\%) & 1213 (90.5\%) & 127 (9.5\%) & & 607 (45.7\%) \\
\hline \multicolumn{4}{|l|}{ WBCs, $\times 10^{9}$} & 0.137 & \\
\hline$<6.9$ & 1376 (51.2\%) & 1289 (93.7\%) & 87 (6.3\%) & & 677 (50.9\%) \\
\hline$\geq 6.9$ & 1309 (48.8\%) & 1207 (92.2\%) & $102(7.8 \%)$ & & 652 (49.1\%) \\
\hline \multicolumn{4}{|l|}{ Neutrophils, $\times 10^{9}$} & 0.001 & \\
\hline$<4.2$ & 1356 (50.5\%) & 1283 (94.6\%) & $73(5.4 \%)$ & & 691 (52.0\%) \\
\hline$\geq 4.2$ & 1329 (49.5\%) & 1213 (91.3\%) & 116 (8.7\%) & & 638 (48.0\%) \\
\hline \multicolumn{6}{|l|}{$H G B, g / L$} \\
\hline$<145$ & 1379 (51.4\%) & 1264 (91.7\%) & $115(8.3 \%)$ & 0.007 & 758 (57.0\%) \\
\hline$\geq 145$ & 1306 (48.6\%) & 1232 (94.3\%) & $74(5.7 \%)$ & & 571 (43.0\%) \\
\hline \multicolumn{4}{|l|}{ Platelets, $\times 10^{9}$} & 0.013 & \\
\hline$<229$ & 1343 (50.0\%) & 1265 (94.2\%) & $78(5.8 \%)$ & & $638(48.0 \%)$ \\
\hline$\geq 229$ & 1342 (50.0\%) & 1231 (91.7\%) & $111(8.3 \%)$ & & 691 (52.0\%) \\
\hline \multicolumn{4}{|l|}{$\mathrm{ALT}, \mathrm{U} / \mathrm{L}$} & 0.392 & \\
\hline$<22.2$ & 1345 (50.1\%) & 1256 (93.4\%) & 89 (6.6\%) & & 725 (54.6\%) \\
\hline
\end{tabular}


Table 1 Associations between the clinical and laboratory characteristics of the patients and SMAD as indicated by the chi-square test or Fisher's exact test (Continued)

\begin{tabular}{|c|c|c|c|c|c|}
\hline$\geq 22.2$ & 1340 (49.9\%) & $1240(92.5 \%)$ & $100(7.5 \%)$ & & $604(45.4 \%)$ \\
\hline AST, U/L & & & & 0.092 & \\
\hline$<21$ & $1366(50.9)$ & 1281 (93.8\%) & $85(6.2 \%)$ & & $675(50.8 \%)$ \\
\hline$\geq 21$ & $1319(49.1 \%)$ & 1215 (92.1\%) & $104(7.9 \%)$ & & $654(49.2 \%)$ \\
\hline$A L P, U / L$ & & & & $<0.001$ & \\
\hline$<70$ & 1357 (50.5\%) & 1304 (96.1\%) & $53(3.9 \%)$ & & $744(56.0 \%)$ \\
\hline$\geq 70$ & $1328(49.5 \%)$ & 1192 (89.8\%) & $136(10.2 \%)$ & & $585(44.0 \%)$ \\
\hline $\mathrm{LDH}, \mathrm{U} / \mathrm{L}$ & & & & $<0.001$ & \\
\hline$<172.2$ & $1344(50.1 \%)$ & $1287(95.8 \%)$ & $57(4.2 \%)$ & & 706 (53.1\%) \\
\hline$\geq 172.2$ & 1341 (49.9\%) & $1209(90.2 \%)$ & $132(9.8 \%)$ & & $623(46.9 \%)$ \\
\hline$A L B, g / L$ & & & & 0.003 & \\
\hline$<44.9$ & 1351 (50.3\%) & $1236(91.5 \%)$ & $115(8.5 \%)$ & & $576(43.3 \%)$ \\
\hline$\geq 44.9$ & 1334 (49.7\%) & $1260(94.5 \%)$ & $74(5.5 \%)$ & & $753(56.7 \%)$ \\
\hline GLB, g/L & & & & 0.507 & \\
\hline$<30.5$ & 1341 (49.9\%) & $1251(93.3 \%)$ & $90(6.7 \%)$ & & $793(59.7 \%)$ \\
\hline$\geq 30.5$ & $1344(50.1 \%)$ & $1245(92.6 \%)$ & 99 (7.4\%) & & $536(40.3 \%)$ \\
\hline Cholesterol, mmol/L & & & & 0.054 & \\
\hline$<5.12$ & 1353 (50.4\%) & $1245(92.0 \%)$ & $108(8.0 \%)$ & & $576(43.3 \%)$ \\
\hline$\geq 5.2$ & 1332 (49.6\%) & 1251 (93.9\%) & $81(6.1 \%)$ & & $753(56.7 \%)$ \\
\hline T lymphocytes, $\times 10^{9}$ & & & & 0.289 & \\
\hline$<1.8$ & $1392(51.8 \%)$ & 1287 (92.5\%) & $105(7.5 \%)$ & & $622(46.8 \%)$ \\
\hline$\geq 1.8$ & $1293(48.2 \%)$ & 1209 (93.5\%) & $84(6.5 \%)$ & & 707 (53.2\%) \\
\hline Monocytes, $\times 10^{9}$ & & & & 0.005 & \\
\hline$<0.4$ & $1385(51.6 \%)$ & 1306 (94.3\%) & 79 (5.7\%) & & 462 (34.8\%) \\
\hline$\geq 0.4$ & 1300 (48.4\%) & 1190 (91.5\%) & $110(8.5 \%)$ & & 867 (65.2\%) \\
\hline Pathology & & & & 0.852 & \\
\hline Undifferentiated & $2592(96.5 \%)$ & 2410 (93.0\%) & $182(7.0 \%)$ & & $1300(97.8 \%)$ \\
\hline Differentiated & $93(3.5 \%)$ & $86(92.5 \%)$ & $7(7.5 \%)$ & & $29(2.2 \%)$ \\
\hline Cranial nerve injury & & & & 0.730 & \\
\hline Absent & $2498(93.0 \%)$ & 2321 (92.9\%) & $177(7.1 \%)$ & & $1234(92.9 \%)$ \\
\hline Present & $187(7.0 \%)$ & 175 (93.6\%) & $12(6.4 \%)$ & & $95(7.1 \%)$ \\
\hline EBV-DNA, copies/ml & & & & $<0.001$ & \\
\hline$<1000$ & 1130 (42.1\%) & 1092 (96.6\%) & $38(3.4 \%)$ & & $526(39.6 \%)$ \\
\hline 1000-9999 & 585 (21.8\%) & 555 (94.9\%) & $30(5.1 \%)$ & & 265 (19.9\%) \\
\hline $10,000-99,999$ & $599(22.3 \%)$ & 555 (92.7\%) & $44(23.3 \%)$ & & 325 (24.5\%) \\
\hline $100,000-999,999$ & $290(10.8 \%)$ & $245(84.5 \%)$ & $45(15.5 \%)$ & & $156(11.7 \%)$ \\
\hline$\geq 1,000,000$ & $81(3.0 \%)$ & $49(60.5 \%)$ & $32(39.5 \%)$ & & $57(4.3 \%)$ \\
\hline T category & & & & 0.804 & \\
\hline 1 & $167(6.2 \%)$ & $158(94.6 \%)$ & 37 (5.4\%) & & $81(6.1 \%)$ \\
\hline 2 & $525(19.6 \%)$ & $488(93.0 \%)$ & $37(7.0 \%)$ & & $328(24.7 \%)$ \\
\hline 3 & $1374(51.2 \%)$ & 1278 (93.0\%) & $96(7.0 \%)$ & & $630(47.4 \%)$ \\
\hline 4 & $619(23.1 \%)$ & $572(92.4 \%)$ & $47(7.6 \%)$ & & $290(21.8 \%)$ \\
\hline N category & & & & $<0.001$ & \\
\hline 0 & 319 (11.9\%) & 312 (97.8\%) & $7(2.2 \%)$ & & $250(18.8 \%)$ \\
\hline
\end{tabular}


Table 1 Associations between the clinical and laboratory characteristics of the patients and SMAD as indicated by the chi-square test or Fisher's exact test (Continued)

\begin{tabular}{|c|c|c|c|c|c|}
\hline 1 & $921(34.3 \%)$ & 887 (96.3\%) & $34(3.7 \%)$ & & $449(33.8 \%)$ \\
\hline 2 & $775(28.9 \%)$ & $697(89.9 \%)$ & 78 (10.1\%) & & $370(27.8 \%)$ \\
\hline 3 & $549(20.4 \%)$ & $494(90.0 \%)$ & $55(10.0 \%)$ & & $243(18.3 \%)$ \\
\hline 4 & $121(4.5 \%)$ & $106(87.6 \%)$ & $15(12.4 \%)$ & & $17(1.3 \%)$ \\
\hline Radiotherapy technique & & & & 0.451 & \\
\hline IMRT +3DCRT & $1341(49.9 \%)$ & $1252(93.4 \%)$ & $89(6.6 \%)$ & & $705(65.9 \%)$ \\
\hline CRT & 1344(51.1\%) & $1244(92.6 \%)$ & $100(7.4 \%)$ & & $624(34.1 \%)$ \\
\hline Treatment method & & & & $P<0.001$ & \\
\hline Radiotherapy & $505(18.8 \%)$ & 481 (95.2\%) & $24(4.8 \%)$ & & $318(24.1 \%)$ \\
\hline CCRT & $1136(42.3 \%)$ & 1086 (95.6\%) & $50(4.4 \%)$ & & $425(32.2 \%)$ \\
\hline $\mathrm{Neo}+$ radiotherapy & $483(18.0 \%)$ & $419(86.7 \%)$ & $64(13.3 \%)$ & & $265(20.1 \%)$ \\
\hline $\mathrm{NeO}+\mathrm{CCRT}$ & $561(20.9 \%)$ & $510(90.9 \%)$ & $51(9.1 \%)$ & & $311(23.5 \%)$ \\
\hline \multicolumn{6}{|l|}{ SMAD } \\
\hline Absent & $2496(93.0 \%)$ & & & & $1231(92.6 \%)$ \\
\hline Present & $189(7 \%)$ & & & & $98(7.4 \%)$ \\
\hline
\end{tabular}

Abbreviations: SMAD skeletal metastasis at time of diagnosis, WBCs white blood cells, $H G B$ hemoglobin, GLB globulin, $A L B$ albumin, $A L T$ alanine transaminase, $A S T$ aspartate transaminase, $A L P$ alkaline phosphatase, $L D H$ lactate dehydrogenase, CRP C-reactive protein, GGT gamma glutamyl transpeptidase, EBV-DNA Epstein-Barr virus DNA, Undifferentiated undifferentiated non-keratinizing carcinoma, Differentiated differentiated carcinoma, CRT conventional radiotherapy, IMRT intensity modulated radiation therapy, $3 D-C R T$ three dimensional conformal radiation therapy, $R T$ radiotherapy, $C C R T$ concurrent radiotherapy, Neo neoadjuvant chemotherapy

The factors associated with significantly poorer SMFS in the univariate Cox regression models were advanced age; elevated LDH, CRP, ALP, monocytes and plasma EBV-DNA; decreased globulin (GLB) and ALB; and advanced clinical $\mathrm{N}$ category. ALP, LDH, CRP, plasma EBV DNA and $\mathrm{N}$ category retained independent prognostic value in multivariate logistic regression. Detailed summaries of the multivariate analyses are shown in Tables 2 and 3.

\section{Nomograms for predicting SMAD and SMFS}

The independent prognostic factors for SMAD and SMFS were used to construct nomograms (Fig. 1). Each variable was assigned a score. By determining the total score for all variables on the total point scale, the probabilities of specific outcomes could be determined by drawing a vertical line from the total score. Plasma EBV DNA copy number was the most important factor for prediction of both SMAD and SMFS.

In the training cohort, the SMAD nomogram had a bootstrap-corrected c-index of 0.83 (95\% CI, 0.78-0.87), significantly higher than the TNM classification (0.73; 95\% CI, 0.70-0.77; $P=0.005)$. The c-index of the nomogram for SMFS $(0.70 ; 95 \% \mathrm{CI}, 0.67-0.74)$ was also significantly higher than the TNM classification (0.59; 95\% CI, 0.56-0.63; $P<0.001)$. In the external validation cohort, the c-index value of the nomogram for SMAD was 0.76 (95\% CI, 0.71-0.79) and 0.61 (95\% CI, 0.550.66) for SMFS; both of which were significantly better than the c-index values for the TNM classification with respect to SMAD $(0.64 ; 95 \%$ CI, $0.60-0.67 ; P<0.001)$ and SMFS $(0.58 ; 95 \% \mathrm{CI}, 0.54-0.63 ; P=0.005)$, respectively (Table 4).

The calibration plots demonstrated good agreement between the nomogram predictions and actual 1-, 3-, and 5-year SMFS rates observed in both the training and the validation cohorts (Fig. 2).

\section{Nomograms for risk stratification}

We determined the cut-off values for the nomogramgenerated scores by which the patients in the training cohort could be stratified into three risk groups. Each group had a distinct prognosis (Additional file 3: Table S2). This stratification could effectively predict SMFS for the three proposed risk groups in both the training and validation cohorts (Fig. 3). The risk stratification even provided significant distinction between the Kaplan-Meier SMFS curves for each of the three risk groups within each TNM stage (Fig. 3).

\section{Discussion}

This is the first study to retrospectively assess a very large number of patients with NPC to evaluate the prognostic value of a wide range of clinical and laboratory parameters in order to establish effective prognostic tools for skeletal metastasis. The nomograms established in this analysis demonstrated superior discriminative ability compared to the TMM classification of the 
Table 2 Associations between the clinical and laboratory characteristics of the patients and SMAD in univariate and multivariate logistic regression analysis

\begin{tabular}{|c|c|c|c|c|c|c|}
\hline \multirow[t]{2}{*}{ Characteristic } & \multicolumn{3}{|c|}{$\underline{\text { Univariate }}$} & \multicolumn{3}{|c|}{ Multivariate } \\
\hline & $\overline{H R}$ & $95 \% \mathrm{Cl}$ & $P$-value & $\overline{H R}$ & $95 \% \mathrm{Cl}$ & $P$-value \\
\hline Age ( $\geq 45$ vs. $<45$ years) & 1.142 & $0.850-1.535$ & 0.379 & & & \\
\hline Gender (Male vs. Female) & 0.623 & $0.410-0.946$ & 0.027 & & & \\
\hline Smoking Status (Present vs. Absent) & 1.139 & $0.993-1.807$ & 0.056 & & & \\
\hline Drinking Status (Present vs. Absent) & 1.038 & $0.655-1.647$ & 0.873 & & & \\
\hline Family history (Present vs. Absent) & 0.907 & $0.649-1.267$ & 0.566 & & & \\
\hline Calcium, mmol/L ( $\geq 2.4$ vs. < 2.4) & 0.987 & $0.734-1.327$ & 0.932 & & & \\
\hline Phosphorus, mmol/L ( $\geq 1.15$ vs. < 1.15) & 0.921 & $0.685-1.239$ & 0.587 & & & \\
\hline Magnesium, $\mathrm{mmol} / \mathrm{L}$ ( $\geq 0.93$ vs. $<0.93$ ) & 0.857 & $0.636-1.154$ & 0.308 & & & \\
\hline CRP, mg/L ( $\geq 1.91$ vs. < 1.91) & 2.167 & $1.583-2.965$ & $<0.001$ & & & \\
\hline WBCs, $\times 10^{9}(\geq 6.9$ vs. $<6.9)$ & 1.252 & $0.931-1.684$ & 0.137 & & & \\
\hline Neutrophils, $\times 10^{9}$ ( $\geq 4.2$ vs. $<4.2$ ) & 1.681 & $1.241-2.276$ & 0.001 & & & \\
\hline HGB, g/L ( $\geq 145$ vs. $<145)$ & 0.660 & $0.488-0.893$ & 0.007 & 0.672 & $0.477-0.948$ & 0.023 \\
\hline Platelets, $\times 10^{9}$ ( $\geq 229$ vs. < 229) & 1.462 & $1.083-1.974$ & 0.013 & & & \\
\hline ALT, U/L ( $\geq 22.2$ vs. $<22.2)$ & 1.138 & $0.846-1.530$ & 0.392 & & & \\
\hline AST, U/L ( $\geq 21$ vs. $<21)$ & 1.290 & $0.958-1.736$ & 0.093 & & & \\
\hline ALP, U/L ( $\geq 70$ vs. $<70)$ & 2.807 & $2.024-3.893$ & $<0.001$ & 2.148 & $1.509-3.056$ & $<0.001$ \\
\hline $\mathrm{LDH}, \mathrm{U} / \mathrm{L}(\geq 172.2$ vs. < 172.2) & 2.465 & $1.789-3.396$ & $<0.001$ & 1.512 & $1.069-2.139$ & 0.019 \\
\hline ALB, g/L ( $\geq 44.9$ vs. $<44.9)$ & 0.631 & $0.466-0.854$ & 0.003 & & & \\
\hline GLB, g/L ( $\geq 30.5$ vs. $<30.5)$ & 1.105 & $0.822-1.486$ & 0.507 & & & \\
\hline Cholesterol, mmol/L ( $\geq 5.12$ vs. < 5.12) & 0.746 & $0.554-1.006$ & 0.055 & & & \\
\hline T lymphocytes, $\times 10^{9}$ ( $\geq 1.8$ vs. $<1.8$ ) & 0.852 & $0.632-1.147$ & 0.290 & & & \\
\hline Monocytes, $\times 10^{9}$ ( $\geq 0.4$ vs. $<0.4$ ) & 1.528 & $1.133-2.062$ & 0.006 & & & \\
\hline Pathology (Differentiated vs. Undifferentiated & 1.078 & $0.492-2.363$ & 0.852 & & & \\
\hline Cranial nerve injury (Absent vs. Present) & 0.899 & $0.491-1.646$ & 0.899 & & & \\
\hline EBV-DNA, copies/ml & & & $<0.001$ & & & $<0.001$ \\
\hline$<1000$ & 1.000 & 1.000 & & 1.000 & 1.000 & \\
\hline 1000-9999 & 1.553 & $0.952-2.534$ & 0.078 & 1.293 & $0.784-2.131$ & 0.314 \\
\hline $10,000-99,999$ & 2.278 & $1.459-3.558$ & $<0.001$ & 1.588 & $0.998-2.530$ & 0.051 \\
\hline 100,000-999,999 & 5.278 & $3.354-8.307$ & $<0.001$ & 3.234 & $1.982-5.279$ & $<0.001$ \\
\hline$\geq 1,000,000$ & 18.767 & $10.822-32.544$ & $<0.001$ & 10.703 & 5.876-19.498 & $<0.001$ \\
\hline T category & & & 0.805 & & & \\
\hline 1 & 1.000 & 1.000 & & & & \\
\hline 2 & 1.331 & $0.629-2.818$ & 0.455 & & & \\
\hline 3 & 1.319 & $0.653-2.663$ & 0.440 & & & \\
\hline 4 & 1.443 & $0.692-3.007$ & 0.328 & & & \\
\hline N category & & & $<0.001$ & & & 0.002 \\
\hline 0 & 1.000 & 1.000 & & 1.000 & 1.000 & \\
\hline 1 & 1.708 & $0.750-3.893$ & 0.202 & 1.292 & $0.559-2.984$ & 0.549 \\
\hline 2 & 4.988 & $2.276-10.933$ & $<0.001$ & 2.924 & $1.304-6.557$ & 0.009 \\
\hline 3 & 4.962 & $2.232-11.035$ & $<0.001$ & 2.299 & $0.996-5.306$ & 0.051 \\
\hline 4 & 6.307 & $2.504-15.887$ & $<0.001$ & 2.606 & $0.983-6.905$ & 0.054 \\
\hline
\end{tabular}

Abbreviations: SMAD skeletal metastasis at the time of diagnosis, WBCs white blood cells, HGB hemoglobin, GLB globulin, $A L B$ albumin, $A L T$ alanine transaminase, AST aspartate transaminase, ALP alkaline phosphatase, $L D H$ lactate dehydrogenase, CRP C-reactive protein, GGT gamma glutamyl transpeptidase, EBV-DNA Epstein-Barr virus DNA, Undifferentiated undifferentiated non-keratinizing carcinoma, Differentiated differentiated carcinoma 
Table 3 Associations between the clinical and laboratory characteristics of the patients and SMFS in univariate and multivariate logistic regression analysis

\begin{tabular}{|c|c|c|c|c|c|c|}
\hline \multirow[t]{2}{*}{ Characteristic } & \multicolumn{3}{|c|}{ Univariate } & \multicolumn{3}{|c|}{ Multivariate } \\
\hline & $\overline{\mathrm{HR}}$ & $95 \% \mathrm{Cl}$ & $\overline{P \text {-value }}$ & $\overline{\mathrm{HR}}$ & $95 \% \mathrm{Cl}$ & $P$-value \\
\hline Age ( $\geq 45$ vs. $<45$ years) & 1.288 & $1.008-1.647$ & 0.043 & & & \\
\hline Gender (Male vs. Female) & 0.867 & $0.635-1.184$ & 0.371 & & & \\
\hline Smoking Status (Present vs. Absent) & 1.120 & $0.871-1.440$ & 0.376 & & & \\
\hline Drinking Status (Present vs. Absent) & 0.911 & $0.615-1.349$ & 0.642 & & & \\
\hline Family history (Present vs. Absent) & 0.831 & $0.627-1.010$ & 0.198 & & & \\
\hline Calcium, mmol/L ( $\geq 2.4$ vs. $<2.4)$ & 0.927 & $0.725-1.186$ & 0.548 & & & \\
\hline Phosphorus, mmol/L ( $\geq 1.15$ vs. < 1.15) & 0.927 & $0.725-1.185$ & 0.545 & & & \\
\hline Magnesium, mmol/L ( $\geq 0.93$ vs. < 0.93) & 0.804 & $0.552-1.172$ & 0.257 & & & \\
\hline CRP, mg/L ( $\geq 1.91$ vs. < 1.91) & 2.092 & $1.618-2.706$ & $<0.001$ & 1.450 & $1.108-1.897$ & 0.007 \\
\hline WBCs, $\times 10^{9}(\geq 6.9$ vs. $<6.9)$ & 1.050 & $0.822-1.342$ & 0.694 & & & \\
\hline Neutrophils, $\times 10^{9}$ ( $\geq 4.2$ vs. < 4.2) & 1.177 & $0.921-1.504$ & 0.193 & & & \\
\hline HGB, g/L ( $\geq 145$ vs. $<145)$ & 0.835 & $0.653-1.068$ & 0.150 & & & 0.023 \\
\hline Platelets, $\times 10^{9}$ ( $\geq 229$ vs. $<229$ ) & 1.134 & $0.887-1.449$ & 0.315 & & & \\
\hline ALT, U/L ( $\geq 22.2$ vs. $<22.2$ ) & 0.971 & $0.760-1.241$ & 0.814 & & & \\
\hline AST, U/L ( $\geq 21$ vs. < 21) & 1.283 & $1.003-1.641$ & 0.047 & & & \\
\hline ALP, U/L ( $\geq 70$ vs. < 70) & 2.023 & $1.570-2.606$ & $<0.001$ & 1.654 & $1.275-2.145$ & $<0.001$ \\
\hline LDH, U/L ( $\geq 172.2$ vs. < 172.2) & 1.951 & $1.514-2.514$ & $<0.001$ & 1.424 & $1.098-1.847$ & $<0.001$ \\
\hline ALB, g/L ( $\geq 44.9$ vs. $<44.9)$ & 0.694 & $0.542-0.889$ & 0.004 & & & \\
\hline GLB, g/L ( $\geq 30.5$ vs. $<30.5)$ & 1.594 & $1.242-2.047$ & $<0.001$ & & & \\
\hline Cholesterol, $\mathrm{mmol} / \mathrm{L}$ ( $\geq 5.12$ vs. $<5.12$ ) & 0.955 & $0.747-1.220$ & 0.710 & & & \\
\hline T lymphocytes, $\times 10^{9}$ ( $\geq 1.8$ vs. $<1.8$ ) & 0.913 & $0.714-1.167$ & 0.468 & & & \\
\hline Monocytes, $\times 10^{9}(\geq 0.4$ vs. $<0.4)$ & 1.431 & $1.118-1.832$ & 0.004 & & & \\
\hline Pathology (Differentiated vs. Undifferentiated & 0.410 & $0.153-1.101$ & 0.077 & & & \\
\hline Cranial nerve injury (Absent vs. Present) & 1.075 & $0.666-1.736$ & 0.767 & & & \\
\hline EBV-DNA, copies/ml & & & $<0.001$ & & & $<0.001$ \\
\hline$<1000$ & 1.000 & 1.000 & & 1.000 & 1.000 & \\
\hline 1000-9999 & 1.955 & $1.349-2.832$ & $<0.001$ & 1.521 & $1.045-2.215$ & 0.029 \\
\hline $10,000-99,999$ & 2.757 & $1.959-3.881$ & $<0.001$ & 1.822 & $1.277-2.601$ & 0.001 \\
\hline $100,000-999,999$ & 4.569 & $3.147-6.631$ & $<0.001$ & 2.706 & $1.829-4.004$ & $<0.001$ \\
\hline$\geq 1,000,000$ & 7.451 & $4.221-13.151$ & $<0.001$ & 4.764 & $1.829-8.533$ & $<0.001$ \\
\hline Treatment method & & & & 0.040 & & \\
\hline Radiotherapy & 1.000 & 1.000 & & & & \\
\hline CCRT & 1.064 & $0.639-1.773$ & 0.811 & & & \\
\hline $\mathrm{Neo}+$ Radiotherapy & 0.188 & $0.834-2.521$ & 0.188 & & & \\
\hline $\mathrm{NeO}+\mathrm{CCRT}$ & 0.752 & $0.426-1.325$ & $<0.001$ & & & \\
\hline Radiotherapy technology (IMRT + 3DCRT vs. CRT) & 0.745 & $0.378-1.471$ & 0.397 & & & \\
\hline T category & & & 0.021 & & & \\
\hline 1 & 1.000 & 1.000 & & & & \\
\hline 2 & 3.190 & $1.269-8.020$ & 0.014 & & & \\
\hline 3 & 3.752 & $1.538-9.157$ & 0.004 & & & \\
\hline 4 & 3.966 & $1.596-9.856$ & 0.003 & & & \\
\hline
\end{tabular}


Table 3 Associations between the clinical and laboratory characteristics of the patients and SMFS in univariate and multivariate logistic regression analysis (Continued)

\begin{tabular}{cllllrr}
\hline N category & & & $<0.001$ & & $<0.001$ \\
0 & 1.000 & 1.000 & & 1.000 & 1.000 & $0.765-2.681$ \\
1 & 1.731 & $0.928-3.230$ & 0.085 & 1.432 & 0.262 \\
2 & 3.017 & $1.638-5.558$ & $<0.001$ & 2.149 & $1.156-3.995$ & 0.016 \\
3 & 5.987 & $3.281-10.925$ & $<0.001$ & 3.613 & $1.947-6.704$ & $<0.001$ \\
4 & 6.310 & $3.079-12.933$ & $<0.001$ & 3.629 & $1.742-7.559$ & 0.001 \\
\hline
\end{tabular}

Abbreviations: SMFS skeletal metastasis-free survival, WBCs white blood cells, HGB hemoglobin, GLB globulin, $A L B$ albumin, $A L T$ alanine transaminase, $A S T$ aspartate transaminase, $A L P$ alkaline phosphatase, $L D H$ lactate dehydrogenase, $C R P$ C-reactive protein, GGT gamma glutamyl transpeptidase, $E B V$ - $D N A$ Epstein-Barr virus DNA, Undifferentiated undifferentiated non-keratinizing carcinoma, Differentiated, differentiated carcinoma

seventh edition of the UICC/AJCC staging system and enabled risk scoring for individual patients. The independent prognostic factors for skeletal metastasis (SMAD, SMFS) included N category, circulating EBV-DNA, LDH, ALP, HGB and CRP; each of these factors has been previously reported to play a vital role in tumor progression or metastasis.

Advanced $\mathrm{N}$ category was significantly associated with skeletal metastasis in this study, which reflects the assumption that the tumor cells responsible for distant

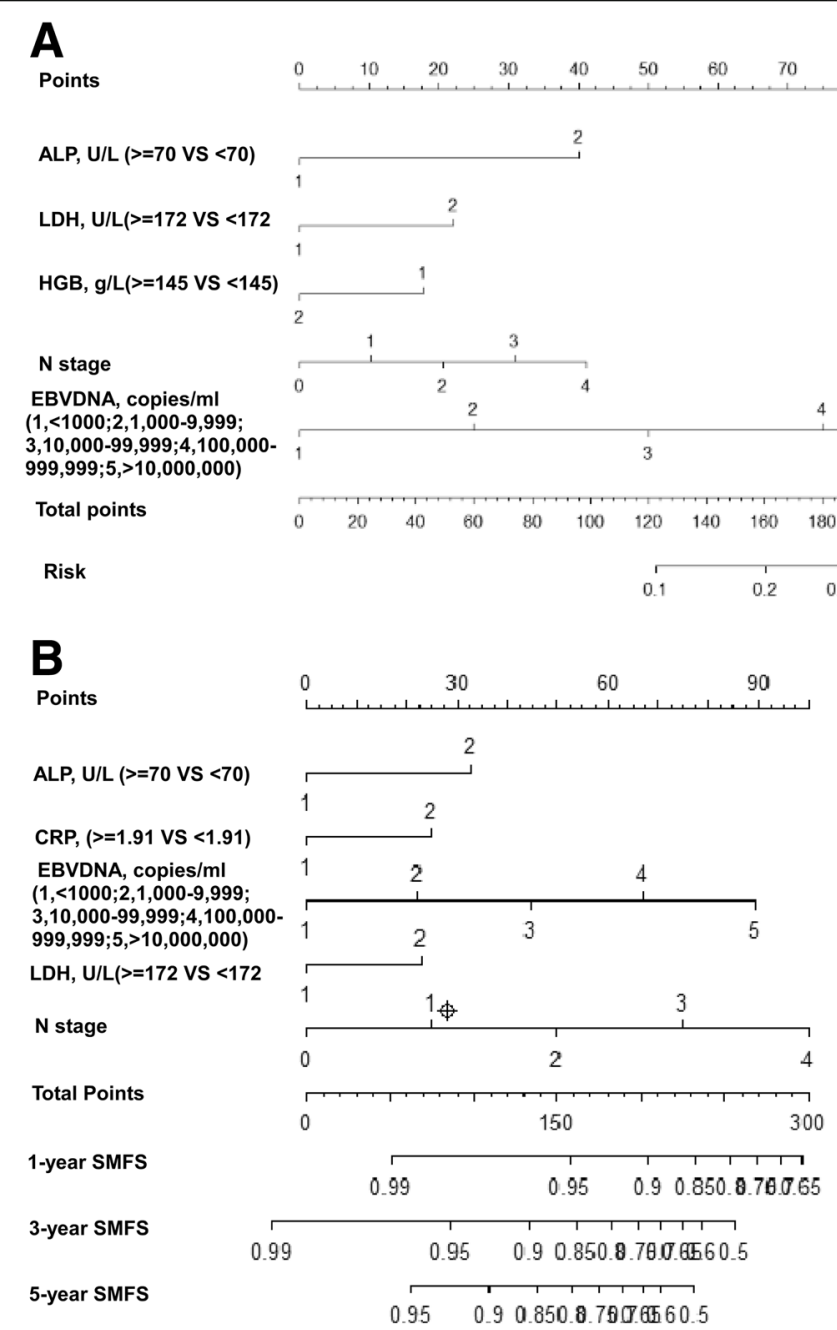

Fig. 1 Nomograms for predicting SMAD (a) and SMFS (b) in NPC. Points refers to the value of each factor included in the nomogram; total points, total points for all factors; 1/3/5-year survival, survival probability based on total points; ALP, alkaline phosphatase; HGB, hemoglobin; LDH, lactate dehydrogenase; CRP, C-reactive protein; EBV, Epstein-Barr virus; SMAD, skeletal metastasis at diagnosis; SMFS, skeletal-metastasis free survival 
Table 4 The c-index values for performance of the multivariate model and the TNM classification for prediction of SMAD and SMFS in the training set and validation set

\begin{tabular}{|c|c|c|c|c|c|c|}
\hline \multirow[t]{2}{*}{ Model } & \multicolumn{3}{|c|}{ Training set } & \multicolumn{3}{|c|}{ Validation set } \\
\hline & C-index & $95 \% \mathrm{Cl}$ & $P$-value & C-index & $95 \% \mathrm{Cl}$ & $P$-value \\
\hline Nomograms (SMAD) & 0.83 & $0.78-0.87$ & 0.005 & 0.76 & $0.71-0.79$ & $<0.001$ \\
\hline TNM classification (SMAD) & 0.73 & $0.70-0.77$ & & 0.64 & $0.60-0.67$ & \\
\hline Nomograms (SMFS) & 0.70 & $0.67-0.74$ & $<0.001$ & 0.61 & $0.55-0.0 .66$ & 0.005 \\
\hline TNM classification (SMFS) & 0.59 & $0.56-0.63$ & & 0.58 & $0.54-0.63$ & \\
\hline
\end{tabular}

Abbreviations: SMAD skeletal metastasis at the time of diagnosis, SMFS skeletal metastasis-free survival

metastasis disseminate from the lymph nodes, rather than the primary tumor. In agreement with our findings, high serum ALP has also previously been reported to be a negative prognostic factor for skeletal metastasis and is used in the clinic to predict the presence of bone metastases in a range of cancers, including lung cancer and prostate cancer [12, 13]. The hydrolase ALP dephosphorylates a variety of molecules. Serum ALP is usually low in healthy individuals, but increases during pregnancy and in patients with bile duct obstruction, kidney disease, hepatocellular carcinoma or bone metastasis [14-18]. Yang et al. reported a high serum LDH level was an independent, unfavorable
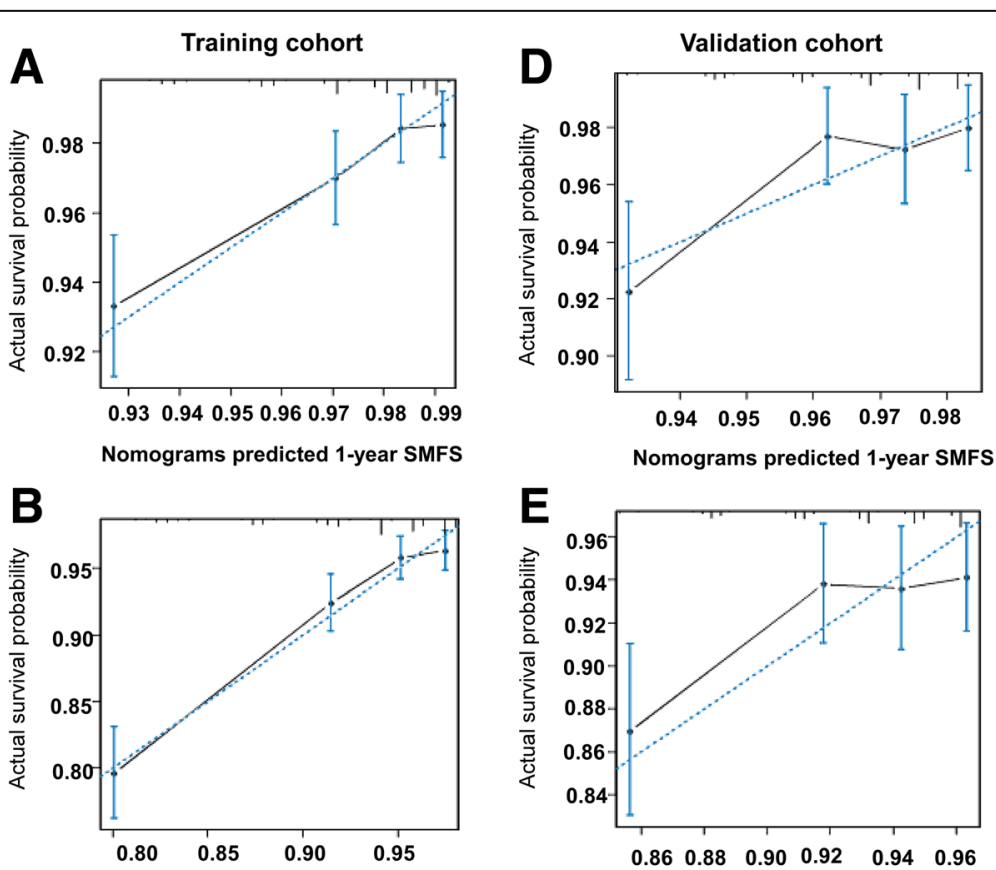

Nomograms predicted 3-year SMFS
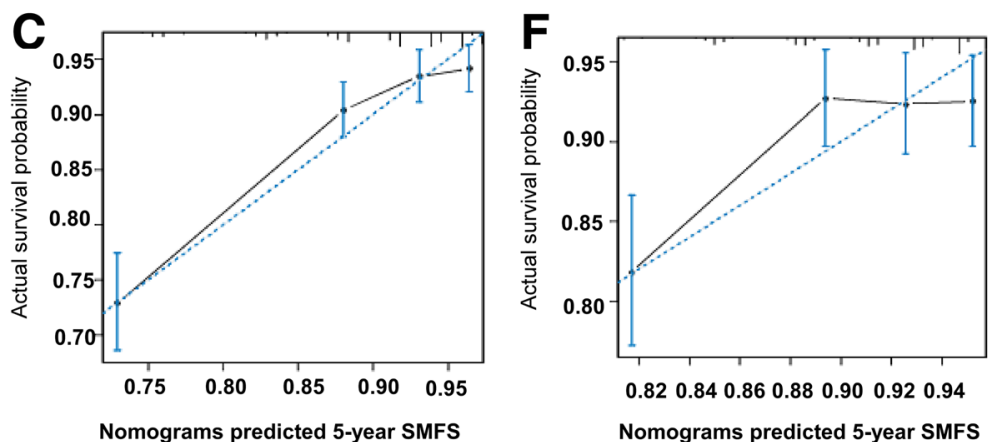

Fig. 2 Calibration plots for SMFS at 1, 3 and 5 years in the training cohort $(\mathbf{a}, \mathbf{b}, \mathbf{c})$ and validation cohort (d, e, $\mathbf{f})$. Nomogram-predicted SMFS is plotted on the $x$-axis; actual rates of SMFS are plotted on the $y$-axis. The dashed lines along the 45-degree line through the origin represent the perfect calibration models in which the predicted probabilities are identical to the actual probabilities. SMFS, skeletal-metastasis free survival 

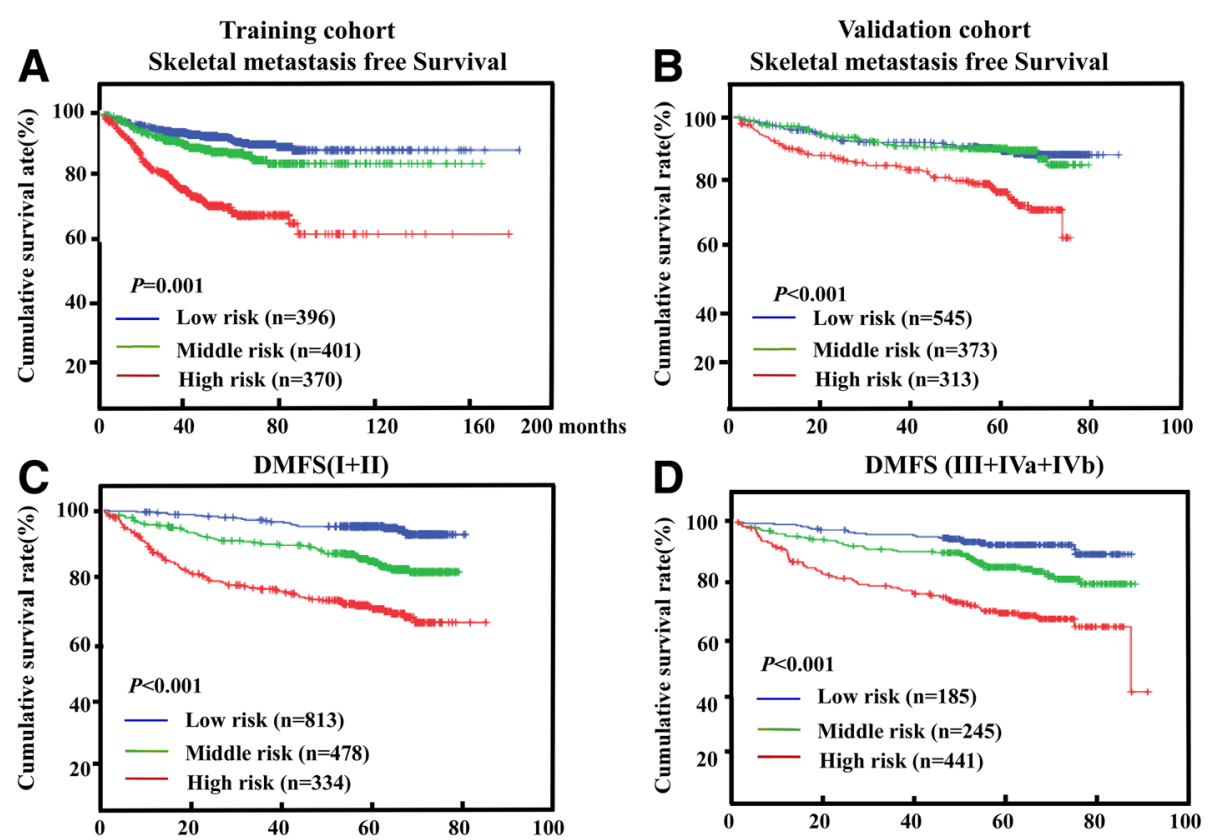

Fig. $\mathbf{3}$ Kaplan-Meier curves of SMFS for the three risk group stratifications. Nomogram risk group stratifications for the 33 and 66 percentiles are shown for the training cohort $(\mathbf{a}, \mathbf{c})$ and validation cohort $(\mathbf{b}, \mathbf{d})$. SMFS, skeletal-metastasis free survival

risk factor for overall survival (OS) and distant-metastasis free survival (DMFS) in non-metastatic NPC [19]. This study provides the first evidence that high serum LDH is an independent prognostic factor for skeletal metastasis in NPC. Rapid tumor cell proliferation initiates anaerobic glycolysis to produce energy, which requires the transformation of pyruvate to lactate by LDH, a key enzyme of glycolysis [20]. In addition, increased LDH levels lead to a low extracellular $\mathrm{pH}$ and activate the hypoxia-inducible factor (HIF) pathway, which is well-recognized to promote tumor growth, aggressiveness and distant metastasis [21-25].

In the regions where NPC is endemic, EBV infection is associated with an increased risk of NPC, and plasma EBV DNA is a useful prognostic marker in both early and advanced NPC [26, 27]. The present study indicates that circulating EBV DNA is also an independent prognostic factor for skeletal metastasis in NPC. Leung et al. reported that the EBV DNA cutoff value of 4000 copies $/ \mathrm{mL}$ could categorize patients with early-stage NPC into a high-risk subgroup (with similar survival outcomes to patients with stage III disease) and a lowrisk subgroup (with similar survival outcomes to stage I disease) [28]. A previously-established nomogram for disease-free survival (DFS) revealed incorporation of plasma EBV DNA increased the C-index compared to the model that did not include EBV DNA [29]. In further confirmation of its prognostic value, plasma EBV DNA was incorporated as a significant factor into the prognostic models for SMAD and SMFS in this study, and resulted in more accurate risk discrimination for individual patients.

Reduced HGB was also an independent prognostic factor for poor SMAD, consistent with the report by Ong et al. [30]. Anemia is more common in patients with advanced stage disease and/or a poor performance status, both of which are associated with a higher probability of skeletal metastasis in NPC. Elevated CRP has been associated with advanced tumor classification, bone invasion and lymph node metastasis in NPC [31]. Similarly, CRP moderately enhanced the predictive ability of the SMFS nomogram in this study. The link between inflammation and cancer is well-recognized; prolonged exposure to proinflammatory cytokines may eventually result in the induction of CRP synthesis and is considered to be a prognostic factor in NPC [32, 33]. In the future, improving nutrition status, inflammatory status and immune function could potentially further improve the clinical outcome of patients with NPC.

The present study has several limitations. First, the time span of data collection was nearly 7 years for the data set. Therefore, the question of whether the nomograms can be applied to patients currently receiving treatment should be asked. However, at our institution, the pathologic examination has not changed during this period of time. Second, patient comorbidities were not assessed. Liu et al. previously reported that comorbidity could affect OS to some extent in NPC [34]. However, the diversity of comorbidities makes it difficult to establish categorized variables and quantify risk. Therefore, the 
prognostic significance of comorbidities should be assessed in future nomogram studies. Finally, whether this nomogram can be applied to younger patients (aged $<18$-yearsold) or patients in areas with a low occurrence of NPC remains to be determined.

In summary, we have developed and externally-validated nomograms to predict SMAD and SMFS based on analyses of a relatively large number of patients with NPC. The nomograms provide significantly better discrimination than the current seventh TNM classification of the AJCC staging system and also enable individualized prognostication of skeletal metastasis. Moreover, the accuracy of the nomograms was validated using large datasets for patients treated at other two institutions. In conclusion, these nomograms represent useful tools for predicting skeletal metastasis, facilitating patient counseling, and providing timely surveillance and clinical assessments.

\section{Conclusion}

This is the first large cohort study to establish a prediction nomogram for skeletal metastasis in non-metastatic NPC; the predictive accuracy of the model was validated in an external cohort.

\section{Additional files}

Additional file1: $\mathrm{R}$ code of the nomograms for SMAD and SMFS in non-metastatic NPC after definitive radiotherapy. (DOCX $20 \mathrm{~kb}$ )

Additional file 2: Figure S1. Schematic of patient inclusion and exclusion. (TIFF 30204 kb)

Additional file 3: Table S1. Associations between clinical and laboratory characteristics and SMFS as indicated by the chi-square test or Fisher's exact test. (DOC $174 \mathrm{~kb}$ )

\section{Abbreviations \\ 18-FDG PET/CT: 18F-deoxyglucose positron emission tomography/computed tomography; ALB: Albumin; ALP: Alkaline phosphatase; ALT: Alanine transaminase; AST: Aspartate transaminase; AUC: Area under curve; BS: Bone scintigraphy; CRP: C-reactive protein; DMFS: Distant-metastasis free survival; EBV: Epstein-Barr virus; GLB: Globulin; HGB: Hemoglobin; HIF: Hypoxia-inducible factor; LDH: Lactate dehydrogenase; NPC: Nasopharyngeal carcinoma; OS: overall survival; RT: radiotherapy; SMAD: Skeletal metastasis at initial diagnosis; SMFS: Skeletal metastasis-free survival}

\section{Acknowledgements}

Not applicable

\section{Funding}

There was no funding for this research.

\section{Availability of data and materials}

Raw data was deposited in the Research Data Deposit system (research data deposit number RDDA2017000293, http://www.researchdata.org.cn) of Sun Yat-sen University Cancer and can be obtained from the corresponding authors on reasonable request.

\section{Authors' contributions}

$Y L, X L P$ and $C Y$ made substantial contributions to study conception and design; YL, WY, HSS, HSD, LSB, CHY and PPJ collected the data; YL, WY, and XLP analyzed the data and drafted the manuscript; LSB, CHY and PPJ analyzed the data; $Y L$ gave final approval of the version to be published;
HSD and CY revised it critically for important intellectual content; CY agreed to be accountable for all aspects of the work and ensuring questions related to the accuracy or integrity of this work are appropriately investigated and resolved. All authors (YL, XLP, WY, CHY, HSS, CHY, LSB, PPJ, HSD and CY) have read and approved the final manuscript.

Ethics approval and consent to participate

Ethical approval was obtained from the institution through the respective institutional review boards, which belong to the Ethics Committee of Sun Yat-sen University Cancer Center. All patients provided written informed consent to participate in this study.

\section{Consent for publication}

Not applicable

\section{Competing interests}

The authors declare that they have no competing interests.

\section{Publisher's Note}

Springer Nature remains neutral with regard to jurisdictional claims in published maps and institutional affiliations.

\section{Author details}

${ }^{1}$ Sun Yat-sen University Cancer Center, 651 East Dong Feng Road, Guangzhou 510060, China. ${ }^{2}$ State Key Laboratory of Oncology in Southern China, Guangzhou, China. ${ }^{3}$ Collaborative Innovation Center for Cancer Medicine, Guangzhou, China. ${ }^{4}$ The Six Affiliated Hospital of Sun Yat-sen University, Guangzhou, China. ${ }^{5}$ The First Hospital of Foshan, Foshan, China. ${ }^{6}$ The Fifth Affiliated Hospital of Sun Yat-sen University, Zhuhai, China.

Received: 3 December 2016 Accepted: 28 August 2017

Published online: 06 September 2017

\section{References}

1. Cao SM, Simons MJ, Qian CN. The prevalence and prevention of nasopharyngeal carcinoma in China. Chinese J Cancer. 2011;30(2):114-9.

2. Fong KW, Chua EJ, Chua ET, Khoo-Tan HS, Lee KM, Lee KS, Sethi VK, Tan BC, Tan TW, Wee J, et al. Patient profile and survival in 270 computer tomography-staged patients with nasopharyngeal cancer treated at the Singapore General Hospital. Ann Acad Med Singap. 1996;25(3):341-6.

3. Heng DM, Wee J, Fong KW, Lian LG, Sethi VK, Chua ET, Yang TL, Khoo Tan HS, Lee KS, Lee KM, et al. Prognostic factors in 677 patients in Singapore with nondisseminated nasopharyngeal carcinoma. Cancer. 1999;86(10):1912-20.

4. Tan EH, Khoo KS, Wee J, Fong KW, Lee KS, Lee KM, Chua ET, Tan T, Khoo-Tan $\mathrm{HS}$, Yang TL, et al. Phase II trial of a paclitaxel and carboplatin combination in Asian patients with metastatic nasopharyngeal carcinoma. Ann Oncol. 1999; 10(2):235-7.

5. Fandi A, Bachouchi M, Azli N, Taamma A, Boussen H, Wibault P, Eschwege F, Armand JP, Simon J, Cvitkovic E. Long-term disease-free survivors in metastatic undifferentiated carcinoma of nasopharyngeal type. J Clin Oncol. 2000;18(6):1324-30.

6. Algra PR, Bloem JL, Tissing H, Falke TH, Arndt JW, Verboom LJ. Detection of vertebral metastases: comparison between MR imaging and bone scintigraphy. Radiographics. 1991;11(2):219-32.

7. Liu FY, Chang JT, Wang HM, Liao CT, Kang CJ, Ng SH, Chan SC, Yen TC. [18F]fluorodeoxyglucose positron emission tomography is more sensitive than skeletal scintigraphy for detecting bone metastasis in endemic nasopharyngeal carcinoma at initial staging. J Clin Oncol. 2006;24(4):599-604.

8. Tsuya A, Kurata T, Tamura K, Fukuoka M. Skeletal metastases in non-small cell lung cancer: a retrospective study. Lung cancer. 2007;57(2):229-32.

9. Sun JM, Ahn JS, Lee S, Kim JA, Lee J, Park YH, Park HC, Ahn MJ, Ahn YC, Park K. Predictors of skeletal-related events in non-small cell lung cancer patients with bone metastases. Lung cancer. 2011;71(1):89-93.

10. Sekine I, Nokihara $H$, Yamamoto $N$, Kunitoh $H$, Ohe $Y$, Tamura T. Risk factors for skeletal-related events in patients with non-small cell lung cancer treated by chemotherapy. Lung cancer. 2009;65(2):219-22.

11. Delea T, Langer C, McKiernan J, Liss M, Edelsberg J, Brandman J, Sung J, Raut M, Oster G. The cost of treatment of skeletal-related events in patients with bone metastases from lung cancer. Oncology. 2004;67(5-6):390-6.

12. Min JW, Um SW, Yim JJ, Yoo CG, Han SK, Shim YS, Kim YW. The role of whole-body FDG PET/CT, Tc 99m MDP bone scintigraphy, and serum 
alkaline phosphatase in detecting bone metastasis in patients with newly diagnosed lung cancer. J Korean Med Sci. 2009;24(2):275-80.

13. Schindler F, Lajolo PP, Pinczowski H, Fonseca FL, Barbieri A, Massonetto LH, Katto FT, Del Giglio A. Bone and total alkaline phosphatase for screening skeletal metastasis in patients with solid tumours. European J Cancer Care. 2008;17(2):152-6.

14. Bashiri A, Katz O, Maor E, Sheiner E, Pack I, Mazor M. Positive placental staining for alkaline phosphatase corresponding with extreme elevation of serum alkaline phosphatase during pregnancy. Arch Gynecol Obstet. 2007;275(3):211-4.

15. Al Mamari S, Djordjevic J, Halliday JS, Chapman RW. Improvement of serum alkaline phosphatase to $<1.5$ upper limit of normal predicts better outcome and reduced risk of cholangiocarcinoma in primary sclerosing cholangitis. J Hepatol. 2013;58(2):329-34.

16. Damera S, Raphael KL, Baird BC, Cheung AK, Greene T, Beddhu S. Serum alkaline phosphatase levels associate with elevated serum C-reactive protein in chronic kidney disease. Kidney Int. 2011;79(2):228-33.

17. Lu Y, Lu Q, Chen HL. Diagnosis of primary liver cancer using lectin affinity chromatography of serum alkaline phosphatase. J Exp Clin Cancer Res. 1997;16(1):75-80.

18. Sonpavde G, Pond GR, Berry WR, de Wit R, Armstrong AJ, Eisenberger MA, Tannock IF. Serum alkaline phosphatase changes predict survival independent of PSA changes in men with castration-resistant prostate cancer and bone metastasis receiving chemotherapy. Urol Oncol. 2012; 30(5):607-13.

19. Yang L, Hong S, Wang Y, He Z, Liang S, Chen H, He S, Wu S, Song L, Chen Y. A novel prognostic score model incorporating CDGSH iron sulfur Domain2 (CISD2) predicts risk of disease progression in laryngeal squamous cell carcinoma. Oncotarget. 2016;7(16):22720-32

20. Warburg O. On the origin of cancer cells. Science. 1956;123(3191):309-14.

21. Axelson H, Fredlund E, Ovenberger M, Landberg G, Pahlman S. Hypoxiainduced dedifferentiation of tumor cells-a mechanism behind heterogeneity and aggressiveness of solid tumors. Semin Cell Dev Biol. 2005;16(4-5):554-63.

22. Colgan SM, Mukherjee S, Major P. Hypoxia-induced lactate dehydrogenase expression and tumor angiogenesis. Clin Colorectal Cancer. 2007;6(6):442-6.

23. Maxwell PH. The HIF pathway in cancer. Semin Cell Dev Biol. 2005;16(4-5): 523-30.

24. Rofstad EK. Microenvironment-induced cancer metastasis. Int J Radiat Biol. 2000;76(5):589-605.

25. Stubbs M, McSheehy PM, Griffiths JR, Bashford CL. Causes and consequences of tumour acidity and implications for treatment. Mol Med Today. 2000;6(1): 15-9.

26. McDermott AL, Dutt SN, Watkinson JC. The aetiology of nasopharyngeal carcinoma. Clinical otolaryngology and allied sciences. 2001;26(2):82-92.

27. Lo YM, Chan AT, Chan LY, Leung SF, Lam CW, Huang DP, Johnson PJ. Molecular prognostication of nasopharyngeal carcinoma by quantitative analysis of circulating Epstein-Barr virus DNA. Cancer Res. 2000;60(24):6878-81.

28. Leung SF, Zee B, Ma BB, Hui EP, Mo F, Lai M, Chan KC, Chan LY, Kwan WH, Lo YM, et al. Plasma Epstein-Barr viral deoxyribonucleic acid quantitation complements tumor-node-metastasis staging prognostication in nasopharyngeal carcinoma. J Clin Oncol. 2006;24(34):5414-8.

29. Tang LQ, Li CF, Li J, Chen WH, Chen QY, Yuan LX, Lai XP, He Y, Xu YX, Hu DP, et al. Establishment and Validation of Prognostic Nomograms for Endemic Nasopharyngeal Carcinoma. J Natl Cancer Inst. 2016;108(1).

30. Ong YK, Heng DM, Chung B, Leong SS, Wee J, Fong KW, Tan T, Tan EH. Design of a prognostic index score for metastatic nasopharyngeal carcinoma. Eur J Cancer. 2003;39(11):1535-41.

31. Chen HH, Chen IH, Liao CT, Wei FC, Lee LY, Huang SF. Preoperative circulating C-reactive protein levels predict pathological aggressiveness in oral squamous cell carcinoma: a retrospective clinical study. Clin Otolaryngol. 2011;36(2):147-53.

32. Balkwill F, Mantovani A. Cancer and inflammation: implications for pharmacology and therapeutics. Clin Pharmacol Ther. 2010;87(4):401-6.

33. Allin $\mathrm{KH}$, Nordestgaard BG. Elevated C-reactive protein in the diagnosis, prognosis, and cause of cancer. Crit Rev Clin Lab Sci. 2011;48(4):155-70.

34. Liu H, Chen QY, Guo L, Tang LQ, Mo HY, Zhong ZL, Huang PY, Luo DH, Sun R, Guo X, et al. Feasibility and efficacy of chemoradiotherapy for elderly patients with locoregionally advanced nasopharyngeal carcinoma: results from a matched cohort analysis. Radiat Oncol. 2013;8:70.

\section{Submit your next manuscript to BioMed Central and we will help you at every step:}

- We accept pre-submission inquiries

- Our selector tool helps you to find the most relevant journal

- We provide round the clock customer support

- Convenient online submission

- Thorough peer review

- Inclusion in PubMed and all major indexing services

- Maximum visibility for your research

Submit your manuscript at www.biomedcentral.com/submit
Biomed Central 